\title{
The IOC relative energy deficiency in sport clinical assessment tool (RED-S CAT)
}

\author{
Margo Mountjoy, ${ }_{1}$ Jorunn Sundgot-Borgen, ${ }^{2}$ Louise Burke, ${ }^{3}$ \\ Susan Carter, ${ }^{4}$ Naama Constantini, ${ }_{1}^{5}$ Constance Lebrun, ${ }^{6}$ \\ Nanna Meyer, ${ }^{7}$ Roberta Sherman, ${ }^{8}$ Kathrin Steffen, ${ }^{2,9}$ \\ Richard Budgett, ${ }^{9}$ Arne Ljungqvist, ${ }^{9}$ Kathryn Ackerman ${ }^{10}$
}

In April 2014, the International Olympic Committee (IOC) published a Consensus Statement in the British Journal of Sports Medicine (BJSM) entitled "Beyond the Female Athlete Triad - Relative Energy Deficiency in Sport (RED-S)". To assist sports medicine professionals working in clinical sports medicine with the practical screening and management of the RED-S athlete, the IOC authors have developed a Clinical Assessment Tool - the RED-S CAT.

It is well known that the utility of scientific knowledge is limited at a practical

\footnotetext{
${ }^{1}$ Department of Family Medicine, Michael G. DeGroote School of Medicine, McMaster University, Hamilton, Ontario, Canada; 'Department of Sports Medicine, The Norwegian School of Sport Sciences, Oslo, Norway; ${ }^{3}$ Department of Sports Nutrition, Australian Institute of Sport, Belconnen, Australia; ${ }^{4}$ University of Northern Colorado, University of Colorado Medical School, Colorado, USA; ${ }^{5}$ Orthopedic Department, HadassahHebrew University Medical Center, Jerusalem, Israel; ${ }^{6}$ Department of Family Medicine, Faculty of Medicine \& Dentistry, and Glen Sather Sports Medicine Clinic, University of Alberta, Edmonton, Alberta, Canada; ${ }^{7}$ Health Sciences Department, University of Colorado, Colorado Springs, Colorado, USA; ${ }^{8}$ The Victory Program at McCallum Place, St. Louis, Missouri, USA; ${ }^{9}$ IOC Medical and Scientific Department, Lausanne, Switzerland; ${ }^{10}$ Divisions of Sports Medicine and Endocrinology, Boston Children's Hospital, Neuroendocrine Unit. Massachusetts General Hospital, Harvard Medical School, USA
}

Correspondence to Margo Mountjoy, Department of Family Medicine, Michael G. DeGroote School of Medicine, McMaster University, Hamilton, Ontario, Canada; mmsportdoc@mcmaster.ca4444 level and that implementing effective interventions in the real life sport setting are challenging. As in all areas of medicine, there is now attention in sports medicine to the growing field of knowledge translation. Effective translation of the science into practical usable formats are necessary to ensure that athlete care is both evidence based and effective.

Sports medicine clinicians utilise guiding principles and various models to assist with the medical management and harm minimisation in their course of their care of athletes. The RED-S 'Red Light - Yellow Light

- Green Light' Risk Assessment and Return to Play (RTP) models are designed to take a complex clinical assessment and RTP decision making process and integrate them into a functional model that is both simple to understand by the athlete and the clinician, and is relatively easy to implement in the 'real world'. Effective sports medicine models are designed with latitude to accommodate the interpretation of an athlete's unique situation by the treating clinician, acknowledgement of mitigating factors, ongoing monitoring of the individual, and continual re-evaluation of the model. The RED-S 'Red Light - Yellow Light - Green Light' Risk Assessment and Return to Play models were developed with this flexibility to allow clinicians in the field the ability to adapt the model to their particular athlete situation. These models enable the treating clinicians to apply their knowledge of the sport-specific demands and case-specific parameters, combined with their clinical experience, within the flexible parameters of the model. The models were developed to be adaptable for both males and females. The endpoints identified as red light "high risk" criteria and the yellow light "caution" criteria all apply to both male and female athletes except for the two endpoints related to the menstrual cycle. These models have been implemented successfully since 2012 at the competitive level, for all ages and sport disciplines of athletes at the Norwegian Olympic Training Center. The IOC authors recommend that the RED-S conceptual models should be integrated into performance nutrition educational approaches, as they offer an opportunity for athletes and coaches to understand the broad scope of issues related to suboptimal eating practices.

To facilitate this recommendation, the IOC authors have developed a RED-S Clinical Assessment Tool (RED-S CAT) modelled after the Sport Concussion Assessment Tool (SCAT-3), which is widely used in clinical practice. Utilisation of the RED-S CAT will assist clinicians in the field with the screening of athlete populations at risk and the management of return to play decisions of RED-S athletes. Like the original SCAT, the RED-S CAT is designed to facilitate clinical practice and to encourage further research and validation. It is expected that the RED-S CAT will evolve over time as the body of science in the field grows.

The RED-S CAT should be implemented globally to facilitate and improve the medical management of both male and female athletes with RED-S.

- The RED-S CAT clinical assessment tool is available on our website http://bjsm.bmj.com.

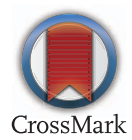

To cite Mountjoy M, Sundgot-Borgen J, Burke L, et al. Br J Sports Med 2015;49:1354.

Published Online First 20 April 2015

Br J Sports Med 2015;49:1354. doi:10.1136/bjsports-2015-094873 